\title{
Mycobacterium riyadhense sp. nov., a non- tuberculous species identified as Mycobacterium tuberculosis complex by a commercial line-probe assay
}

\author{
Correspondence \\ Jakko van Ingen \\ jakko.van.ingen@rivm.nl
}

\author{
Jakko van Ingen, ${ }^{1,2}$ Sahal A. M. Al-Hajoj, ${ }^{3}$ Martin Boeree, ${ }^{2}$ \\ Fahad Al-Rabiah, ${ }^{4}$ Mimount Enaimi, ${ }^{1}$ Rina de Zwaan, ${ }^{1}$ Enrico Tortoli, ${ }^{5}$ \\ Richard Dekhuijzen ${ }^{2}$ and Dick van Soolingen ${ }^{1}$
${ }^{1}$ National Mycobacteria Reference Laboratory, National Institute for Public Health and the Environment, Bilthoven, The Netherlands
${ }^{2}$ Department of Pulmonary Diseases, Radboud University Nijmegen Medical Center, Nijmegen, The Netherlands
${ }^{3}$ Department of Comparative Medicine, King Faisal Specialist Hospital and Research Centre, Riyadh, Saudi Arabia
${ }^{4}$ Department of Medicine, King Faisal Specialist Hospital and Research Centre, Riyadh, Saudi Arabia Hospital, Florence, Italy \\ ${ }^{5}$ Regional Reference Center for Mycobacteria, Microbiology and Virology Laboratory, Careggi
}

\begin{abstract}
A non-chromogenic, slowly growing Mycobacterium strain was isolated from a maxillary sinus lavage from a symptomatic patient in Riyadh, Saudi Arabia. It was initially identified as a member of the Mycobacterium tuberculosis complex by a commercial line-probe assay. Its 16S rRNA, $h s p 65$ and $r p o B$ gene and $16 \mathrm{~S}-23 \mathrm{~S}$ internal transcribed spacer sequences were unique; phylogenetic analysis based on the 16S rRNA gene sequence groups this organism close to Mycobacterium szulgai and Mycobacterium malmoense. Its unique biochemical properties and mycolic acid profile support separate species status. We propose the name Mycobacterium riyadhense sp. nov. to accommodate this strain. The type strain is NLA000201958 $\left(=\mathrm{CIP} 109808^{\top}=\mathrm{DSM}\right.$ $\left.45176^{\top}\right)$.
\end{abstract}

A 19-year-old male reported to the otolaryngology department of the King Faisal Hospital with pain and swelling of the left side of his face with protrusion of left eye after blunt trauma. A computed tomography scan of the sinuses was performed, which revealed a tumour in the left maxillary sinus. The tumour involved the nasal septum, extended into the left orbit and infiltrated the medial and inferior rectus as well as the optic nerve. A chest radiograph revealed no abnormalities. A lavage of both maxillary sinuses was performed for diagnosis. Mycobacterial cultures were performed on Lowenstein-Jensen medium

\section{Abbreviation: ITS, internal transcribed spacer.}

The GenBank/EMBL/DDBJ accession numbers for the sequences of the 16S rRNA, 16S-23S ITS, rpoB, hsp65, esat- 6 and cfp-10 genes of strain NLA000201958' ${ }^{\top}$ are EU274642, EU274643, EU274644, EU921671, EU552926 and EU552927.

Phylogenetic trees based on 16S-23S internal transcribed spacer and hsp65 sequences of selected mycobacterial species are available as supplementary material with the online version of this paper. and yielded an isolate with unusual colony morphology after 3 weeks of incubation at $36{ }^{\circ} \mathrm{C}$.

The patient was presumed to have bone tuberculosis and started a 9-month anti-tuberculosis regimen. The patient improved both clinically and radiologically. He has not suffered a relapse since. The isolated strain was sent to the Dutch National Mycobacteria Reference Laboratory (RIVM, Bilthoven, Netherlands) for identification, as part of a second-line quality control programme. Identification of the strain was first attempted using three commercial line-probe assays, Hain GenoType MTBC and GenoType AS/CM (Hain Lifescience GmbH) and INNO-LiPA MYCOBACTERIA v2 (Innogenetics NV), all used according to the manufacturers' instructions.

To obtain identification to the species level, we sequenced the 16S rRNA gene, 16S-23S internal transcribed spacer (ITS) and $r p o B$ and $h s p 65$ genes, using previously described approaches (Springer et al., 1996; Roth et al., 1998; Kim 
et al., 1999; Telenti et al., 1993). The DNA sequences obtained were compared with the RIDOM (Ribosomal Differentiation of Medical Microorganisms; http://rdna. ridom.de) and GenBank (National Center for Biotechnology Information; http://www.ncbi.nlm.nih.gov) databases.

To establish the presence of a region of difference 1 (RD1)like element and the DNA sequences of the esat- 6 and $c f p$ 10 genes, we used primers from a previous study (Arend et al., 2005).

The most commonly investigated biochemical and phenotypic features [colony morphology, ability to grow at temperatures ranging from 24 to $45{ }^{\circ} \mathrm{C}$, niacin accumulation, nitrate reduction, $\beta$-glucosidase, Tween 80 hydrolysis, 3 day arylsulfatase, urease, tellurite reduction, $68{ }^{\circ} \mathrm{C}$ and semiquantitative catalase, growth rate, pigmentation, growth on MacConkey agar and tolerance to $\mathrm{NaCl}$, thiophene-2-carboxylic hydrazide $(\mathrm{TCH})$, oleate and $p$ nitrobenzoic acid and to $10 \mu \mathrm{g}$ thiacetazone $\mathrm{ml}^{-1}, 1 \mu \mathrm{g}$ hydroxylamine $\mathrm{ml}^{-1}$ and $500 \mu \mathrm{g}$ isoniazid $\mathrm{ml}^{-1}$, all three in Middlebrook 7H10 agar] as well as HPLC analysis of cell-wall mycolic acids were tested by the Regional Reference Center for Mycobacteria (Careggi Hospital, Florence, Italy) using standard procedures described previously (Kent \& Kubica, 1985; CDC, 1996).

Drug susceptibility testing was performed using the 25-well agar-dilution method (van Klingeren et al., 2007). We included isoniazid, rifampicin, rifabutin, ethambutol, clarithromycin, ciprofloxacin, cycloserine, prothionamide, amikacin, clofazimine and streptomycin in the test panel.

Applying the Hain GenoType MTBC assay, a non-specific reaction was noted with hybridization of the $M$. tuberculosis complex band only (banding pattern 1, 2, 3). The InnoLipa MYCOBACTERIA v2 line-probe assay yielded a Mycobacterium genus probe reaction, though no speciesspecific result. The Hain GenoType CM (Common Mycobacteria) kit identified the strain as Mycobacterium tuberculosis complex based on a band 1, 2, 3, 10, 16 pattern. The supplementary AS kit identified the strain as a nonspecified Mycobacterium species, with banding pattern 1, 2, 3, 12. Sequencing of the full 23S rRNA gene, the assay's target, established its identity as a non-tuberculous mycobacterium (Table 1). Based on these findings, IS6110 and IS1081 RFLP were performed to confirm the identity of the strain as $M$. tuberculosis (van Soolingen et al., 1993). No IS6110 or IS1081 element copies could be demonstrated (results not shown).

The sequencing results are listed in Table 1; the 16S rRNA gene, ITS and $r p o B$ and $h s p 65$ gene sequences were all unique. Sequencing of the full $16 \mathrm{~S}$ rRNA gene identified the bacterium as a Mycobacterium szulgai-like species (Table 1). The 16S rRNA gene sequence was aligned with those of reference strains of the closest related mycobacteria using CLUSTAL_X software (Thompson et al., 1997). The resulting topology and tree, inferred by neighbourjoining and visualized using the MEGA software package (Tamura et al., 2007), were evaluated by bootstrap analyses based on 1000 resamplings (Fig. 1). The tree was rooted with Nocardia abscessus ATCC BAA $-279^{\mathrm{T}}$ as an outgroup. Similar multisequence alignments and trees were created based on the ITS and hsp65 sequences (Supplementary Fig. $\mathrm{S} 1$, available in IJSEM Online).

We were able to amplify an RD1 region, including esat-6 (270 bp) and $c f p-10(251 \mathrm{bp})$ genes. Results of sequence comparisons for both genes are recorded in Table 1.

Table 1. Sequence comparisons between strain NLA000201958 ${ }^{\top}$ and its closest relatives

Where no strain is indicated, multiple highly similar sequences are deposited in GenBank.

\begin{tabular}{|c|c|c|}
\hline \multirow[t]{2}{*}{ Gene/region } & \multicolumn{2}{|c|}{ Most similar sequences } \\
\hline & BLAST & RIDOM \\
\hline 16S rRNA gene (full) & $\begin{array}{l}\text { M. malmoense ( } 99 \%), \text { M. szulgai (99\%), M. } \\
\text { bohemicum ( } 98 \%)\end{array}$ & $\begin{array}{l}\text { M. szulgai DSM } 44166^{\mathrm{T}}(99.1 \%) \text {, M. intracellulare } \\
\text { ATCC } 35770(98.4 \%) \text {, M. intracellulare ATCC } 35772 \\
(98.4 \%) \text {, M. haemophilum ATCC } 29548^{\mathrm{T}}(98.4 \%)\end{array}$ \\
\hline 16S-23S ITS (273 bp) & $\begin{array}{l}\text { M. szulgai (91\%), M. kansasii (91\%), } M . \\
\text { marinum }(91 \%)\end{array}$ & $\begin{array}{l}\text { M. kansasii DSM } 44162^{\mathrm{T}}(92 \%) \text {, M. gastri DSM } 43505^{\mathrm{T}} \\
(91 \%), \text { M. marinum DSM } 44344^{\mathrm{T}}(90 \%)\end{array}$ \\
\hline $23 \mathrm{~S}$ rRNA gene (full) & $\begin{array}{l}\text { M. kansasii (97\%), M. avium (97\%), M. ulcerans } \\
\text { agy99 (97\%) }\end{array}$ & \\
\hline rpoB (472 bp) & $\begin{array}{l}\text { M. avium } 104(93 \%) \text {, M. paratuberculosis k10 } \\
(93 \%), \text { M. tuberculosis } \mathrm{H}_{37 \mathrm{Rv}^{\mathrm{T}}}(91 \%)\end{array}$ & \\
\hline hsp65 (421 bp) & $\begin{array}{l}\text { M. genavense DSM } 44424^{\mathrm{T}}(95 \%), \text { M. bohemicum } \\
\text { CIP } 105811^{\mathrm{T}}(95 \%) \text {, M. malmoense CIP } 105775^{\mathrm{T}} \\
(95 \%)\end{array}$ & \\
\hline esat-6 (270 bp) & $\begin{array}{l}\text { M. kansasii }(89 \%), \text { M. tuberculosis } \mathrm{H}_{37 \mathrm{Rv}^{\mathrm{T}}}(87 \%) \text {, } \\
\text { M. szulgai }(85 \%)\end{array}$ & \\
\hline$c f p-10(251 \mathrm{bp})$ & $\begin{array}{l}\text { M. tuberculosis } \mathrm{H}_{37 \mathrm{Rv}^{\mathrm{T}}}(88 \%), \text { M. marinum } \mathrm{M} \\
(85 \%), \text { M. ulcerans }(84 \%)\end{array}$ & \\
\hline
\end{tabular}




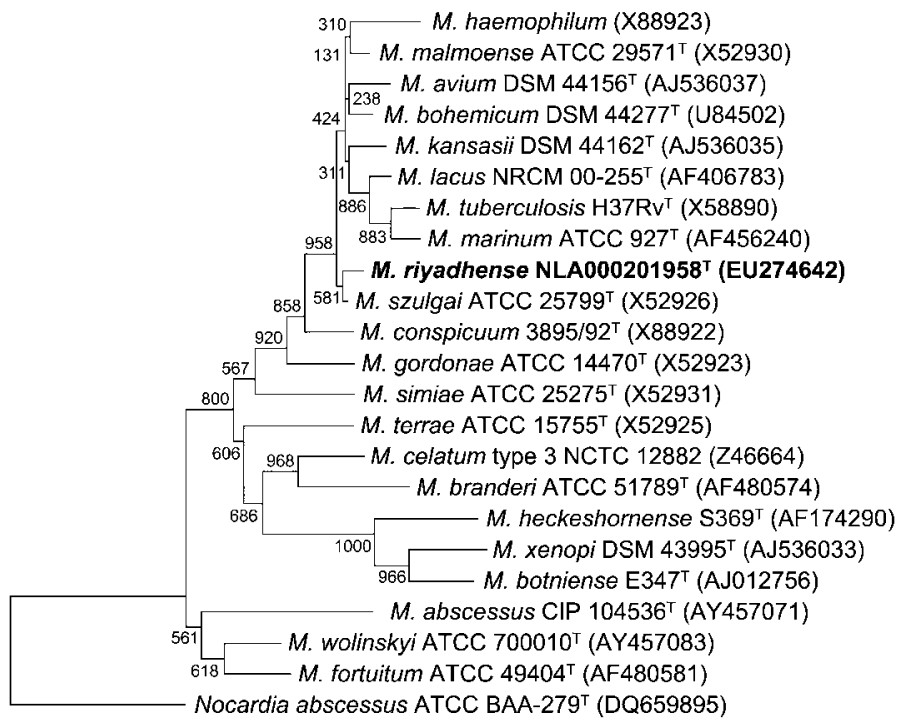

Fig. 1. Phylogenetic relationships of strain

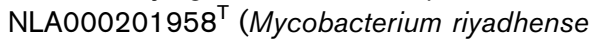
sp. nov.) and related species of Mycobacterium, based on 16S rRNA gene sequences. Neighbour-joining tree created and bootstrapped 1000 times with CLUSTAL_X (Thompson et al., 1997) and visualized with MEGA (Tamura et al., 2007). Bootstrap values are indicated at nodes.
On Middlebrook 7H10, Ogawa and Stonebrink media, the strain produced small, rough, non-pigmented colonies after 28 days of incubation at $36^{\circ} \mathrm{C}$. After 3 days of exposure to light at ambient temperature, no pigmentation was observed. Growth on Middlebrook 7H10 agar was only observed at 24, 30 and $36{ }^{\circ} \mathrm{C}$. Optimal growth occurred at $36{ }^{\circ} \mathrm{C}$. Colony morphology on Middlebrook 7H10 agar was similar at all temperatures.

Generally, the biochemical profile of the isolate is unique, although it shares characteristics with $M$. szulgai and Mycobacterium malmoense (Marks et al., 1972). The isolate was negative for niacin accumulation, heat-stable catalase $\left(\mathrm{pH} 7,68^{\circ} \mathrm{C}\right), \beta$-glucosidase, tellurite reduction, growth on MacConkey agar and tolerance to p-nitrobenzoic acid, hydroxylamine and oleic acid, but positive for nitrate reduction, semiquantitative catalase, Tween 80 hydrolysis, 3 day arylsulfatase and urease activity and tolerance to $\mathrm{TCH}$, thiacetazone and isoniazid. A comparison with profiles of M. szulgai and M. malmoense is recorded in Table 2 .

HPLC revealed a pattern characterized by a single, narrow, late-emerging cluster of peaks. A similar profile is presented by a limited number of mycobacteria (Mycobacterium brumae, M. fallax, M. triviale and $M$. tuberculosis complex), with none of them fully overlapping the pattern of the strain characterized here (Fig. 2). We

Table 2. Biochemical identification results of strain $\mathrm{NLA000201958}^{\top}$ and related species

$+/-$, Strain-dependent reaction.

\begin{tabular}{|c|c|c|c|}
\hline Test & Strain NLA000201958 ${ }^{\mathrm{T}}$ & M. szulgai & M. malmoense \\
\hline Nitrate reduction & + & + & - \\
\hline $68^{\circ} \mathrm{C}$ Catalase & - & + & $+1-$ \\
\hline Catalase $>45 \mathrm{~mm}$ & + & + & - \\
\hline$\beta$-Glucosidase & - & $+1-$ & - \\
\hline Tween 80 hydrolysis & + & $+1-$ & + \\
\hline Tellurite reduction & - & $+1-$ & + \\
\hline 3 day Arylsulfatase & + & + & - \\
\hline Urease & + & + & - \\
\hline Pigmentation & Absent & Photochromogen & Absent \\
\hline Colony morphology & Rough & Smooth/rough & Smooth \\
\hline Growth at $25^{\circ} \mathrm{C}$ & + & + & $+1-$ \\
\hline \multicolumn{4}{|l|}{ Tolerance to: } \\
\hline p-Nitrobenzoic acid & - & + & + \\
\hline Isoniazid $\left(500 \mu \mathrm{g} \mathrm{ml}^{-1}\right)^{\star}$ & + & $+1-$ & + \\
\hline Hydroxylamine $\left(1 \mu \mathrm{g} \mathrm{ml}^{-1}\right)^{*}$ & - & - & $+1-$ \\
\hline
\end{tabular}

${ }^{\star}$ In Middlebrook 7H10 agar. 


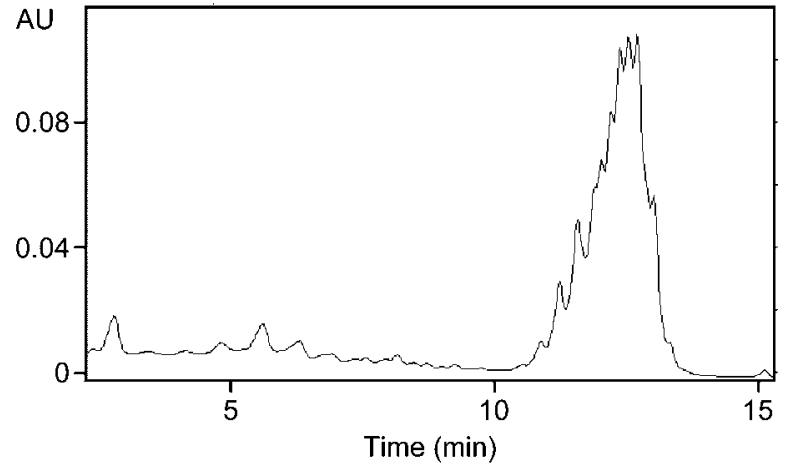

Fig. 2. Mycolic acid pattern of strain NLA000201958 ${ }^{\top}(M$. riyadhense sp. nov.) obtained by HPLC analysis. The single, narrow, late-emerging cluster of peaks is similar, though not identical, to results for $M$. brumae, M. fallax, $M$. triviale and $M$. tuberculosis complex.

used the HPLC mycobacterium library (http://www.mycobactoscana.it/English.htm) for this comparison.

Drug susceptibility testing (minimal inhibitory concentrations given in $\mu \mathrm{g} \mathrm{ml}^{-1}$ ) revealed in vitro resistance to amikacin (10) and $p$-aminosalicylate $(>1)$, intermediate susceptibility to isoniazid (1) and susceptibility to rifampicin (0.2), rifabutin $(<0.2)$, ethambutol (5), clarithromycin $(<2)$, ciprofloxacin $(2)$, cycloserine $(20)$, prothionamide $(<1)$, clofazimine $(<0.5)$ and streptomycin $(5)$.

Although identification of $M$. tuberculosis complex is not the main use of the GenoType CM test, false-positive results may lead to incorrect diagnoses of tuberculosis and unwarranted treatment. Aside from the identification as $M$. tuberculosis by a line-probe assay, which sparked this study, both the molecular and HPLC analyses of the novel strain suggest a phylogenetic relationship with M. tuberculosis; the presence of an RD1 region strengthens this assumption. Its identity as a non-tuberculous mycobacterium is easily proven by sequencing of the $16 \mathrm{~S}$ rRNA, $r p o B, h s p 65$ or RD1 genes and the absence of IS6110 and IS1081 elements. The novel strain seems closely related to M. szulgai, based on $16 S$ rRNA gene and ITS sequences (Fig. 1 and Supplementary Fig. S1a), although it is related more distantly to M. szulgai based on $r p o B$ and hsp65 sequences (Table 1 and Supplementary Fig. S1b). Its non-chromogenicity, however, is distinct from the photochromogenic M. szulgai.

Retrospectively, this isolate seems clinically relevant, based on its isolation from a normally sterile body site and the symptomatic improvement of the patient after 9 months of tuberculosis therapy (Griffith et al., 2007). This suggests that the novel strain was the causative agent of this patient's disease.

Both the close phylogenetic relationship with species such as M. szulgai and Mycobacterium kansasii, which are among the most pathogenic non-tuberculous mycobacteria (Griffith et al., 2007; van Ingen et al., 2008), and the presence of an RD1 region with esat- 6 and $c f p-10$ genes, which is a virulence factor in M. tuberculosis (Lewis et al., 2003), add to our view that this strain is a human pathogen. On the basis of the data presented here, we describe a novel species to accommodate strain NLA000201958 ${ }^{\mathrm{T}}$, for which we propose the name Mycobacterium riyadhense sp. nov.

\section{Description of Mycobacterium riyadhense sp. nov.}

Mycobacterium riyadhense (ri.ya.dhen'se. N.L. neut. adj. riyadhense after Riyadh, capital of the Kingdom of Saudi Arabia and origin of the patient from whom the type strain was isolated).

Slowly growing, non-tuberculous mycobacterium that produces rough, white colonies after 28 days of incubation at $36{ }^{\circ} \mathrm{C}$; growth is slower at 25 and $30{ }^{\circ} \mathrm{C}$ and no growth occurs at $42{ }^{\circ} \mathrm{C}$. Incorrectly identified as M. tuberculosis complex by the Hain GenoType CM assay. Negative for niacin accumulation, heat-stable catalase, $\beta$-glucosidase, tellurite reduction, growth on MacConkey agar and tolerance to $p$-nitrobenzoic acid, hydroxylamine and oleic acid, but positive for nitrate reduction, semiquantitative catalase, Tween 80 hydrolysis, arylsulfatase and urease activity and tolerance to $\mathrm{TCH}$, thiacetazone and isoniazid. Readily identifiable by its unique rRNA gene sequences.

The type strain is NLA000201958 ${ }^{\mathrm{T}}\left(=\mathrm{CIP} 109808^{\mathrm{T}}=\mathrm{DSM}\right.$ $45176^{\mathrm{T}}$ ), recovered from maxillary sinus lavage fluid.

\section{References}

Arend, S. M., de Haas, P. E. W., Leyten, E., Rosenkrands, I., Rigouts, L., Andersen, P., Mijs, W., van Dissel, J. T. \& van Soolingen, D. (2005). ESAT-6 and CFP-10 in clinical versus environmental isolates of Mycobacterium kansasii. J Infect Dis 191, 1301-1310.

CDC (1996). Standardized method for HPLC identification of mycobacteria. Atlanta, GA: Centers for Disease Control and Prevention, US Department of Health and Human Services, Public Health Service.

Griffith, D. E., Aksamit, T., Brown-Elliot, B. A., Catanzaro, A., Daley, C., Gordin, F., Holland, S. M., Horsburgh, R., Huitt, G. \& other authors (2007). An official ATS/IDSA statement: diagnosis, treatment, and prevention of nontuberculous mycobacterial diseases. Am J Respir Crit Care Med 175, 367-416.

Kent, P. T. \& Kubica, G. P. (1985). Public Health Mycobacteriology. A Guide for the Level III Laboratory. Atlanta, GA: US Department of Health and Human Services.

Kim, B.-J., Lee, S.-H., Lyu, M.-A., Kim, S.-J., Bai, G.-H., Kim, S.-J., Chae, G.-T., Kim, E.-C., Cha, C.-Y. \& Kook, Y.-H. (1999). Identification of mycobacterial species by comparative sequence analysis of the RNA polymerase gene (rpoB). J Clin Microbiol 37, 1714-1720.

Lewis, K. N., Liao, R., Guinn, K. M., Hickey, M. J., Smith, S., Behr, M. A. \& Sherman, D. R. (2003). Deletion of RD1 from Mycobacterium tuberculosis mimics bacille Calmette-Guerin attenuation. J Infect Dis 187, 117-123. 
Marks, J., Jenkins, P. A. \& Tsukamura, M. (1972). Mycobacterium szulgai - a new pathogen. Tubercle 53, 210-214.

Roth, A., Fischer, M., Hamid, M. E., Michalke, S., Ludwig, W. \& Mauch, H. (1998). Differentiation of phylogenetically related slowly growing mycobacteria based on 16S-23S rRNA gene internal transcribed spacer sequences. J Clin Microbiol 36, 139-147.

Springer, B., Stockman, L., Teschner, K., Roberts, G. D. \& Bottger, E. C. (1996). Two-laboratory collaborative study on identification of mycobacteria: molecular versus phenotypic methods. J Clin Microbiol 34, 296-303.

Tamura, K., Dudley, J., Nei, M. \& Kumar, S. (2007). MEGA4: molecular evolutionary genetics analysis (MEGA) software version 4.0. Mol Biol Evol 24, 1596-1599.

Telenti, A., Marchesis, F., Balz, M., Bally, F., Bottger, E. C. \& Bodmer, T. (1993). Rapid identification of mycobacteria to the species level by polymerase chain reaction and restriction enzyme analysis. J Clin Microbiol 31, 175-178.
Thompson, J. D., Gibson, T. J., Plewniak, F., Jeanmougin, F. \& Higgins, D. G. (1997). The CLUSTAL_X windows interface: flexible strategies for multiple sequence alignment aided by quality analysis tools. Nucleic Acids Res 25, 4876-4882.

van Ingen, J., Boeree, M. J., de Lange, W. C. M., de Haas, P. E. W., Dekhuijzen, P. N. R. \& van Soolingen, D. (2008). Clinical relevance of Mycobacterium szulgai in the Netherlands. Clin Infect Dis 46, 1200-1205.

van Klingeren, B., Dessens-Kroon, M., van der Laan, T., Kremer, K. \& van Soolingen, D. (2007). Drug susceptibility testing of Mycobacterium tuberculosis complex using a high throughput, reproducible, absolute concentration method. J Clin Microbiol 45, 2662-2668.

van Soolingen, D., de Haas, P. E. W., Hermans, P. W. M., Groenen, P. M. A. \& van Embden, J. D. A. (1993). Comparison of various repetitive DNA elements as genetic markers for strain differentiation and epidemiology of Mycobacterium tuberculosis. J Clin Microbiol 31, 1987-1995. 